Andrea Malits*

\title{
Infrastrukturentwicklung für digitale Editionen am Beispiel der Universität Zürich: Herausforderungen, Erfahrungen und Perspektiven
}

https://doi.org/10.1515/bfp-2020-0023

Zusammenfassung: Für die nachhaltige Verfügbarkeit von Forschungsdaten im Allgemeinen und von digitalen Editionen im Besonderen fehlt es in der Schweiz nach wie vor an Infrastrukturen. Neben technischen Lösungen gelten Koordination und ein verlässliches Netzwerk von Dienstleistungen als Gelingensvoraussetzung für die nachhaltige Verfügbarkeit von Daten. Im Jahr 2014 publizierte der Schweizerische Nationalfonds (SNF) neue Fördermöglichkeiten für digitale Editionsprojekte im Rahmen der Infrastrukturförderung und initiierte damit Strukturbildungsprozesse auf lokaler und auch nationaler Ebene. $\mathrm{Zu}$ den wichtigsten Voraussetzungen einer möglichen Förderung zählte entsprechend die Vorlage eines Konzepts zur Gewährleistung der langfristigen Verfügbarkeit der digitalen Edition. Die Frage der Sicherstellung der digitalen Langzeitverfügbarkeit im Rahmen eines Konzepts adressierte auch Hochschulen und Gedächtnisinstitutionen bzw. deren Rolle und Verantwortlichkeit. Vor diesem Hintergrund schlossen sich vier Editionsprojekte der Universität Zürich (UZH), die Zentralbibliothek Zürich (ZB) sowie die auf Forschungs-IT spezialisierte Abteilung S3IT der UZH zusammen und erarbeiteten ein Kooperationskonzept. Der Artikel beleuchtet diese neuen Kooperationsstrukturen, schildert den Status quo und skizziert mögliche nächste Schritte auf dem Weg zu einer lokal und national koordinierten Infrastrukturlösung für digitale Editionen.

Schlüsselwörter: Digitale Editionen, Infrastrukturförderung, Langzeitverfügbarkeit, SNF

Research Data Infrastructure Development for Digital Editions Using the Example of the University of Zurich: Challenges, Experiences, and Prospects

Abstract: Sustainable infrastructure solutions for research data and particularly for digital editions are still missing in Switzerland. It is generally assumed that in order to be successful and sustainable data infrastructures should be based on coordinated networks of dedicated service pro-

*Kontaktperson: Dr. Andrea Malits, andrea.malits@hbz.uzh.ch viders. The Swiss National Science Foundation (SNSF) invited researchers responsible for current or planned digital editions to participate in a call in 2014. That call included the announcement, that as of 2017, the SNSF would support edition projects only within the scope of the infrastructure funding. Researchers were therefore required to think about the sustainability of the digital data generated within their projects. This was the starting point for a new cooperation, since Higher Education Institutions and libraries were requested to get involved as well. Therefore, at UZH a plan was devised on how the research projects, the Central Library, and the department for Service and Support for Science IT (S3IT) would collaborate. This paper outlines the new cooperation forms, describes the status quo and discusses possible further steps towards a sustainable data infrastructure for digital editions.

Keywords: Digital editions, infrastructure funding, sustainability, SNSF

Für die Langzeitverfügbarkeit von Forschungsdaten fehlen in der Schweiz nach wie vor tragfähige Lösungen. Dies gilt im besonderen Maße auch für digitale Editionen. Zur Gewährleistung einer optimalen Zugänglichkeit zu digitalen Editionen sollten zusätzlich zu den digitalisierten Quellen sowie den Editionsdaten sowohl die Präsentationsoberfläche der Online-Edition als auch die entsprechenden Suchund Visualisierungsfunktionalitäten weiterhin erhalten bleiben. Die bis anhin vorherrschende Einzelprojektförderung und die gängigen Selbstorganisationsprozesse der Forschung können nicht sicherstellen, dass nach Ablauf der individuellen Projektförderung digitale Editionen weiterhin zugänglich bleiben. Die Sicherstellung der digitalen Langzeitarchivierung von Forschungsdaten ist weit mehr als eine technische Herausforderung. Bereits 2011 hielten Aschenbrenner und Neuroth in einem grundlegenden Aufsatz über die Situation des Dateninfrastrukturaufbaus in Deutschland fest, dass „die Fachwissenschaftler sowie die weiteren Akteure (Infrastruktureinrichtungen wie Rechenzentren und Bibliotheken) in einem organisatorischen Gesamtkonzept sinnvoll einzubeziehen“ seien, um die strukturellen Rahmenbedingungen für einen dauerhaf- 
ten Betrieb von Dateninfrastrukturen sicherzustellen. ${ }^{1}$ Fünf Jahre später monierte der Rat für Informationsinfrastrukturen in Deutschland erneut eine nach wie vor zersplitterte, wenig koordinierte Infrastrukturlandschaft und hält fest, dass ein „Umsteuern“ notwendig sei, ${ }^{2}$ um eine funktionierende nationale Dateninfrastruktur sicherzustellen:

„Die anstehenden Aufgaben betreffen die Gestaltung eines mehrjährigen Übergangsprozesses: Wissenschaft muss sich in fast allen Bereichen ändern, wenn weltweit aus Forschungsdaten digitale Forschungsdaten und aus Forschungsmethoden digitale Forschungsmethoden werden. Dies gilt auch für Infrastruktureinrichtungen, Repositorien, Archive etc., deren Arbeitsschwerpunkte sich anpassen und entwickeln müssen.“3

Wo steht der Forschungsplatz Schweiz heute bezüglich der Sicherstellung einer nachhaltig funktionierenden Dateninfrastruktur? Welche strukturbildenden Initiativen sind bereits erfolgt? Welche Verantwortlichkeiten für die nachhaltige Verfügbarkeit von digitalen Editionen sind vorhanden? Und in welchem Verhältnis stehen lokale Kooperationen $\mathrm{zu}$ nationalen und internationalen Dateninfrastrukturen? Im Folgenden wird aus Sicht der Universität Zürich dargelegt, wie infolge einer fokussierten Förderinitiative des Schweizerischen Nationalfonds (SNF) neue Kooperationen zwischen Fachwissenschaft, Rechenzentrum/Informatik, Bibliothek und nationalen Infrastrukturprojekten initiiert und z.T. umgesetzt wurden. Exemplarisch wird dabei auf die Edition Johann Caspar Lavater: Historisch kritische Edition ausgewählter Briefwechsel (JCLB) $)^{4}$ Bezug genommen, da sich diese Edition durch einen hohen Digitalisierungsbedarf von Quellenmaterial auszeichnet, was eine zusätzliche Herausforderung darstellt.

\section{SNF-Call initiiert Strukturbildung}

Im Jahr 2014 publizierte der SNF einen Call für die Eingabe von Editionsprojekten, die in der Periode 2017-2020 mit einem Gesamtbetrag von 23,1 Millionen Franken gefördert werden sollten. ${ }^{5}$ Damit verbunden war die Ankündigung, dass digitale Editionen neu im Rahmen der Infrastruktur-

1 Aschenbrenner und Neuroth (2011) 111.

2 RfII (2016) 3.

3 Ebd.

4 Für Informationen zu JCLB siehe auch die entsprechende Webseite: https://www.lavater.uzh.ch/de.html.

5 Siehe dazu den Call: http://www.snf.ch/SiteCollectionDocuments/ call_editionen_d.pdf. förderung des SNF unterstützt würden. Was diese Neuausrichtung konkret bedeutet, blieb zunächst weitgehend offen. Verlangt wurde allerdings - zusätzlich zum wissenschaftlichen Konzept für die Edition - ein „digitales Bearbeitungs- und Publikationskonzept“ mit Überlegungen zur Langzeitarchivierung. Zudem forderte der SNF im Rahmen der Infrastrukturförderung auch eine finanzielle Mitverantwortung von Hochschulen und anderen Organisationen. Aus den genannten Anforderungen ergab sich mit der Entwicklung der Bearbeitungs- und Publikationskonzepte eine Reihe offener Fragen. So galt es zu klären, welche Kosten für IT-Dienstleistungen für die Förderung anrechenbar sein würden. Angesichts der Tatsache, dass Schweizer Hochschulen im Regelfall (noch) keine Infrastrukturen und Dienstleistungen für digitale geisteswissenschaftliche Projekte bereitstellten, musste überlegt werden, inwiefern die Expertise von Datenzentren für Digital Humanities auch aus dem Ausland einbezogen werden könnte, da sich das Schweizer Data and Service Center for the Humanities (DaSCH), mit heutigen Standorten in Basel und Lausanne (letzterer als sogenannter "Satellit“), als Pilotprojekt zu diesem Zeitpunkt erst im Aufbau befand. ${ }^{6}$ Eine weitere wichtige Frage war, ob auch Kosten für die Digitalisierung subsumierbar sein würden. ${ }^{7}$ Besonders herausfordernd war jedoch das vom SNF gewünschte Konzept der Langzeitarchivierung. Wer könnte diese kostenintensive und verantwortungsvolle Aufgabe langfristig übernehmen? War dies als Aufgabe von Hochschulen gedacht? Oder sollte dies nicht eher als nationale Aufgabe verstanden werden? Welche Rolle und Mitverantwortung sollten Hochschulen und Bibliotheken sowie Archive, deren Sammlungen Gegenstand der Editionen sein würden, übernehmen? Diese weitgehend noch ungeklärten Fragen gingen über das gewohnte und bekannte Format des Forschungsantrags für Editionen hinaus und betrafen insbesondere auch Aspekte der Rahmenbedingungen involvierter Institutionen. Der SNF initiierte mit seinem Call für 2017 bei den Editionsprojekten die Bildung neuer Netzwerke und Strukturen, ein Prozess, der bis heute andauert. Im Folgenden werden - mit Fokus auf das Beispiel des Forschungsprojekts JCLB - zentrale Aspekte dieses Strukturfindungsprozesses aufgezeigt.

6 Das DaSCH wird seit 2017 mit nationalem Auftrag als Unternehmung der Schweizerischen Akademie für Geistes- und Sozialwissenschaften (SAGW) betrieben: https://dasch.swiss/about/.

7 Im Publikationskonzept des Forschungsprojekts JCLB war - um ein Mengenbeispiel zu geben - gemäß ersten Abklärungen vorgesehen, die rund 23000 Briefe von und an Lavater in der Zentralbibliothek Zürich (ZB) zwecks Visualisierung des Korrespondentennetzwerks und für die zu edierenden Briefwechsel zu digitalisieren. 


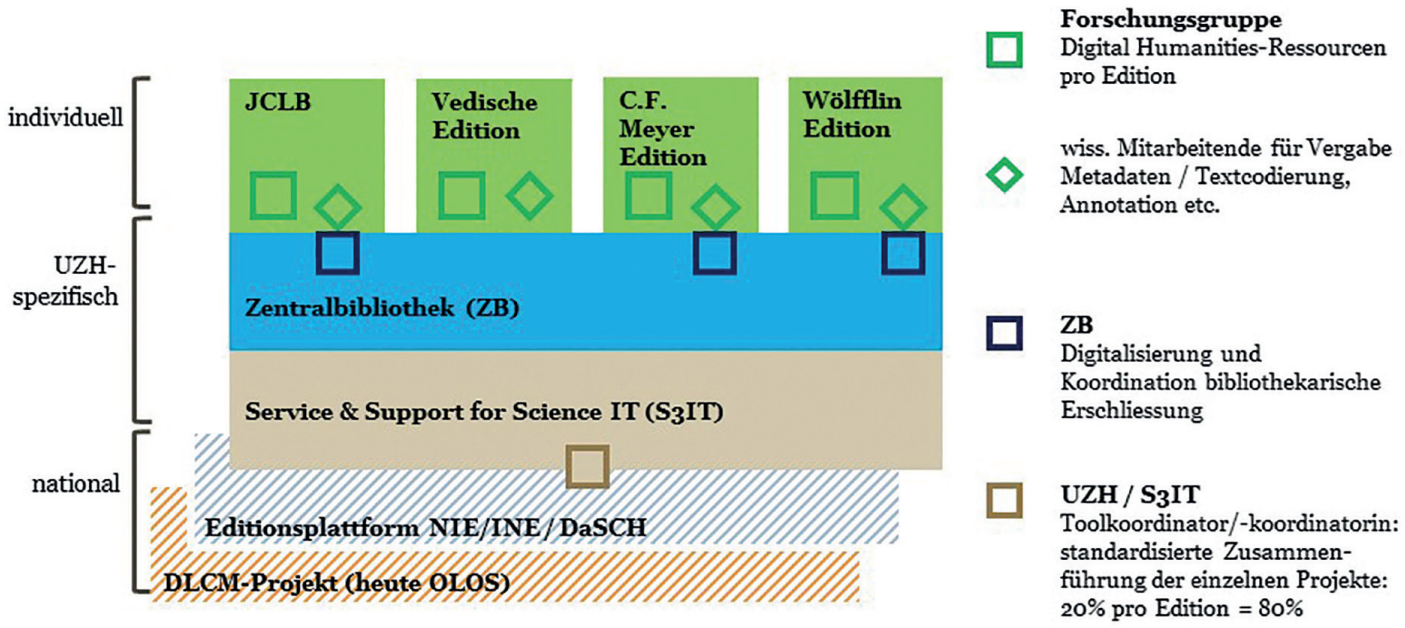

Abb. 1: Konzeptskizze der unterschiedlichen Ebenen der Zusammenarbeit, Grafik A. Malits

\section{Planung lokaler und nationaler Kooperationen}

In der Phase der Erarbeitung der digitalen Bearbeitungsund Publikationskonzepte schlossen sich an der Universität Zürich vier Editionen, die sich am SNF-Call beteiligen wollten, zusammen. Es waren dies die Edition von C.F. Meyers Verlagsbriefwechsel, ${ }^{8}$ die Online-Edition der Paippalada-Rezension des Atharvaveda, ${ }^{9}$ das schon erwähnte Editionsprojekt JCLB sowie Heinrich Wölfflins Gesammelte Werke. ${ }^{10}$ In einer ersten frühen Phase der Erarbeitung der Publikationskonzepte zuhanden des SNF entstanden Modelle und Skizzen, die eine mögliche strukturierte Zusammenarbeit visualisierten, was sich für die Verständigung unter den verschiedenen Partnern als nützlich zeigte. Eine solch enge Kooperation von Forschungsprojekten mit der Bibliothek und der Informatik einerseits sowie mit den für Digital Humanities spezialisierten Datenzentren und Plattformen andererseits war neu. Sie erforderte nebst zahlreichen Konzeptvorschlägen ebenso viele Gespräche, um die Anforderungen und (Dienstleistungs-)Möglichkeiten zu klären. Das Ergebnis war ein Konzept mit den wichtigsten Aufgaben und Verantwortlichkeiten der einzelnen Partner (vgl. Abb. 1).

Das Konzept in Abb. 1 zeigt alle beteiligten Kooperationspartner in Schichten dargestellt: Zuoberst finden sich die einzelnen Editionen mit ihren individuellen Anfor-

8 http://www.cfmeyer.ch/editionsprojekt.

9 Für eine Kurzbeschreibung: https://www.atharvavedapaippalada. uzh.ch/en.html.

10 https://www.woelfflin.uzh.ch/de.html. derungen, Inhalten und Forschungszielen. Das Fundament bilden die nationalen Partner und Plattformen als technische Basis, deren Mandat und Aufgabe die Langzeitarchivierung ist (DaSCH, NIE-INE, DLCM-Projekt, siehe dazu unten im Kapitel zur Langzeitarchivierung). Zwischen diesen beiden Schichten liegen lokale, an der UZH bzw. in Zürich beheimatete Serviceeinheiten wie die auf Science-IT spezialisierte Abteilung S3IT der Universität Zürich (UZH) und die Zentralbibliothek Zürich (ZB). Deren Zuständigkeit ist es, zwischen den einzelnen Editionsprojekten auf der einen Seite und den für die Langzeitarchivierung zuständigen Institutionen auf der anderen Seite zu vermitteln. Dies beinhaltet die flexible Koordination der technischen Umsetzung individueller Editionslösungen einerseits und der verlangten Standards für die Langzeitverfügbarkeit der Daten andererseits.

Die in den einzelnen Schichten eingezeichneten Rhomben und Quadrate symbolisieren Aufgaben- bzw. Stellenprofile, die für die Umsetzung der Editionen inkl. der Anforderung der Langzeitarchivierung benötigt werden. Diese unterschiedlichen Aufgabenprofile, die im Folgenden noch näher erläutert werden, verdeutlichen ein zentrales Resultat der ersten Phase der Strukturfindung. Sie konkretisieren schematisch die Zusammenarbeit unter den verschiedenen beteiligten Partner und bildeten die Basis für die Förderanträge im Rahmen der Infrastrukturförderung durch den SNF.

Das Aufgabenprofil „Digital Humanities“ ist zentral für alle vier Editionen. Dieses Profil beinhaltet - sehr pauschal formuliert - die forschungsbezogenen Aufgaben der Konzeption und Umsetzung der digitalen Editionen.

Für die Digitalisierung sowie die Ersterschließung der Quellenmaterialien wurden die Bibliotheken vorgesehen. 
Im Fall von JCLB war dies die ZB, in deren Bestand das Quellenmaterial für die Edition zum größten Teil liegt. Eine frühe Konzeption der engen Kooperation war daher in beidseitigem Interesse. Die ZB ist Stadt-, Kantons- und Universitätsbibliothek mit Fokus auf Geistes- und Sozialwissenschaften. Für sie bedeutet das Projekt eine Aufarbeitung und prominente Sichtbarmachung einer ihrer zentralen Bestände. Das Forschungsprojekt JCLB gewann über diese Zusammenarbeit Zugang zur ausgezeichneten Digitalisierungsinfrastruktur der ZB. Darüber hinaus versprach die enge Kooperation auch eine Möglichkeit, bestehende Arbeitsprozesse der Digitalisierung und Erschließung mit Vorteil nachzunutzen. Vor dem eigentlichen Projektbeginn wurde daher zwischen der ZB und den Verantwortlichen von JCLB vereinbart, dass die von der Bibliothek erstellten Daten inkl. Metadaten von Anfang an bereits für die Nachnutzung optimiert werden, damit das Editionsprojekt diese Daten über eine Schnittstelle automatisch beziehen und weiterverwenden kann. Vorbild für diese Art der Zusammenarbeit zwischen Bibliothek und Forschungsprojekt war die wegweisende Digitale Edition der Korrespondenz August Wilhelm Schlegels, ${ }^{11}$ wie sie von der Sächsischen Landesbibliothek - Staats- und Universitätsbibliothek - in Dresden (SLUB) in Kooperation mit dem Institut für Neuere deutsche Literatur an der PhilippsUniversität Marburg verantwortet wird. Auf Basis der Erfahrungen dieses Projekts der SLUB wurden für JCLB beim SNF entsprechende Gelder für die bibliothekarische Erschließung von Lavaters Korrespondenz beantragt. Begründet wurde diese Stelle beim SNF mit dem Gewinn von zusätzlichen, über den bibliothekarischen Standard hinausgehenden Metadaten und mit der Erschließung der Briefkonvolute auf Einzelbriefebene.

Eine Besonderheit mit Blick auf die Finanzierung bzw. den Mittelantrag beim SNF stellt das Aufgabenprofil des Toolkoordinators dar. Die vier kooperierenden Editionsprojekte an der UZH einigten sich auf ein gemeinsames Vorgehen für eine solche, bis dahin nicht bekannte Koordinationsstelle. Diese Funktion des Toolkoordinators war als zentrale Koordinationsstelle in allen Fragen der technischen Standardisierung vorgesehen und sollte an der 2014 eingerichteten, auf die technische Forschungsunterstützung spezialisierte Abteilung Service and Support for Science IT (S3IT) angegliedert werden. S3IT unterstützt Forschende der UZH bei der Umsetzung computerbasierter Forschungsaufgaben mit „Beratung, Applikationsunter-

11 https://august-wilhelm-schlegel.de/briefedigital/ bzw. für Informationen zu den Workflows https://august-wilhelm-schlegel.de/brie fedigital/info/workflow. stützung, Zugang zu kompetitiven Cloud- sowie Clusterund Supercomputer-Systemen“. ${ }^{12}$ Die Dienstleistungen sind, wie diese von der Webseite zitierte Beschreibung unschwer erkennen lässt, auf den Bedarf der Life Science ausgerichtet. Dieser Status quo des Serviceangebots bildet allerdings schlicht nur die historische Entwicklung der datenbasierten Forschung ab. Auch hermeneutisch forschende Geisteswissenschaften arbeiten seit geraumer Zeit ebenfalls mit digitalen Methoden und Werkzeugen, im Regelfall allerdings - zumal an der UZH - meist selbstorganisiert und ohne etablierte organisatorische Verankerung und Unterstützung. Der Bedarf der Editionen, insbesondere die Anforderung des SNF, Angaben zur Langzeitarchivierung zu machen, legte es nahe, zu prüfen, ob und welchen Beitrag die S3IT leisten könnte. S3IT verfügte bereits damals über Erfahrung in der Zusammenarbeit mit Forschungsprojekten im Bereich der Life Science und konnte in Anlehnung an die bewährte Praxis in kurzer Zeit ein Modell für die Editionen erarbeiten. Dieses sah vor, dass zur Unterstützung der Editionen für spezialisierte IT-Fragen mit Fokus auf die Langzeitarchivierung eine Stelle benötigt würde, deren Umfang von der betreuten Zahl der Editionen abhängig veranschlagt und vom Aufwand pro Edition berechnet und anteilmäßig in Stellenprozenten pro Edition beim SNF beantragt werden sollte. Die geplante zentrale Ansiedelung der dereinst zuständigen Person bei S3IT sollte für alle beteiligten Editionsprojekte gleichermaßen zur Verfügung stehen. Das Modell vermochte die beteiligten Editionsprojekte zu überzeugen. Sie einigten sich darauf, in ihren individuellen Förderanträgen koordiniert und anteilmäßig die entsprechende Stelle zu beantragen. Dieses gemeinsame Vorgehen wurde mit einem Unterstützungsschreiben von S3IT betreffend die institutionelle Verantwortung für die Laufzeit der Projekte mit der Bereitstellung eines Arbeitsplatzes zugunsten der Editionen geltend gemacht.

Für alle drei der oben skizzierten Aufgabenprofile Profil „Digital Humanities“, „Erschließung“ und „Toolkoordinator" - wurde von den vier Editionen beim SNF im Sinne der Infrastrukturförderung Antrag auf Fördergeld gestellt. Im September 2016 bewilligte der SNF alle vier UZH-Editionen. Für die Förderphase von 2017-2020 waren für das Forschungsprojekt JCLB rund 1,8 Mio. Franken eingeworben worden. ${ }^{13}$ Dabei hatten die Gutachter des

12 Dienstleistungsbeschreibung gemäß Webseite: https://www.zi. uzh.ch/en/teaching-and-research/science-it/.

13 Siehe die vom SNF zur Verfügung gestellte Übersicht: http:// www.snf.ch/SiteCollectionDocuments/Editions_Approved_projects_ of_the_first_call_(September_2016).pdf. 
SNF die in diesem Artikel zur Diskussion stehenden beantragten Aufgabenprofile positiv evaluiert und in die Förderung aufgenommen.

\section{Die Etablierung der neuen Kooperationen}

Für das Aufgabenprofil „Digital Humanities“ entschieden sich die vier Editionen für je unterschiedlichen Lösungen. JCLB hatte bereits in der Antragsphase Kontakt zum Trier Center for Digital Humanities (TCDH) geknüpft. Auf Basis dessen großer Erfahrung, u. a. auch im Rahmen der bereits erwähnten Digitalen Edition der Korrespondenz August Wilhelm Schlegels, konnte nach dem positiven Entscheid durch den SNF das technische Editionskonzept zügig erstellt werden. Das Team von JCLB wurde für die Arbeit mit dem Transkriptionsmodul Transcribo sowie mit dem Metadatenerfassungsprogramm FuD (Forschungsnetzwerk und Datenbank) geschult und die wissenschaftliche Editionsarbeit konnte nach Anpassungen dieser Programme für JCLB bald schon aufgenommen werden.

Bei der aufwändigen Sichtung des Quellenmaterials (der Briefe von und an Lavater) in der ZB wurde bald ersichtlich, dass allein die restauratorische Begutachtung und Behandlung der diversen Briefkonvolute mehr Zeit in Anspruch nehmen dürfte als ursprünglich geplant war. Die Begutachtung unikaler Handschriften hinsichtlich ihres Zustands und allfällig notwendiger bestandserhaltender Maßnahmen hat vor der Digitalisierung zu geschehen, was den straffen Editionsplan herausforderte. Enge Absprachen zwischen JCLB und der ZB für einen Vorgehensplan waren notwendig. Die ZB stellte zudem Sondermittel zur Verfügung, um alle notwendigen restauratorischen Anforderungen mit dem Zeitplan des Forschungsprojekts harmonisieren zu können. Mit dem an der ZB aufgebauten Digitalisierungszentrum und der Publikationsplattform $e$ manuscripta, ${ }^{14}$ welche die ZB mit anderen Schweizer Bibliotheken gemeinsam mitverantwortet, waren exzellente Voraussetzungen für eine Kooperation mit einem Forschungsprojekt wie der digitalen Edition von JCLB gegeben, da für die Verzeichnung und Digitalisierung von historischen Materialien ein bereits etablierter Workflow bestand. Dieser beinhaltet, dass die Metadaten aus dem Bibliothekskatalog in die Publikationsplattform e-manu-

14 https://www.e-manuscripta.ch/. scripta übernommen und über diesen Prozess vollautomatisiert mit den im Digitalisierungszentrum hergestellten Digitalisaten verknüpft werden. Gemäß bibliothekarischem Usus wurden allerdings bis dato für den Bibliothekskatalog im Regelfall keine Metadaten für Einzelbriefe, sondern nur für so genannte Konvolute von Briefen erfasst. Entsprechend waren für das Forschungsprojekt JCLB Anpassungen auf technischer Ebene im System des Visual Library Managers (VLM), dem Verwaltungssystem der Präsentationsplattform e-manuscripta, erforderlich: Mit dieser technischen Neuerung können Briefkonvolute nun in Einzelbriefe strukturiert und mit bibliothekarisch erstellten Metadaten angereichert werden. Die so strukturierten Metadaten, mit dazugehörigen Verlinkungen auf die Digitalisate, werden schließlich über eine Schnittstelle (OAI) automatisiert bezogen und in das Metadatenerfassungsprogramm FuD des Forschungsprojekts importiert. Das wissenschaftliche Team von JCLB verwertet in $\mathrm{FuD}$ bzw. mit Transcribo die Metadaten weiter und transkribiert die Digitalisate, die ebenfalls automatisiert importiert werden. Im Zuge der Kooperation richtete die ZB für die Erstellung der Metadaten, insbesondere jener der Einzelbriefe, auch die vom SNF finanzierte Mitarbeiterstelle für die Laufzeit von drei Jahren ein. Der Rekrutierungsprozess wurde im Laufe des Jahres 2017 gemeinsam von der ZB mit JCLB durchgeführt, der Arbeitsplatz befindet sich in der ZB.

Die Stelle eines Toolkoordinators wurde 2017 in der Abteilung S3IT, befristet bis Ende der Förderphase 2020, eingerichtet. Das Aufgabengebiet dieser neuen Stelle ist schwerpunktmäßig auf die technische Koordination und Standardisierung ausgerichtet, und zwar mit Blick auf die Langzeitarchivierung. Des Weiteren umfasst diese Stelle, abhängig von den Bedürfnissen der einzelnen Editionen, die folgenden Aufgaben: die Evaluation von Software für die standardisierte Bearbeitung der Edition sowie für die Webpublikation, die Konvertierung von spezifischen Projektdaten in generische Ontologien bzw. deren Weiterverwendung in individuellen Webapplikationen, schließlich die Durchführung von Koordinationssitzungen sowie bei Bedarf die Organisation von Schulungen und Workshops für die Editionsprojekte.

\section{Langzeitarchivierung als nationale Aufgabe}

Ebenfalls im Jahr 2016 und damit fast zeitgleich mit den SNF-Anträgen wurde auf nationaler Ebene ein Projekt für eine nationale Plattform für Editionen (NIE-INE) initiiert 


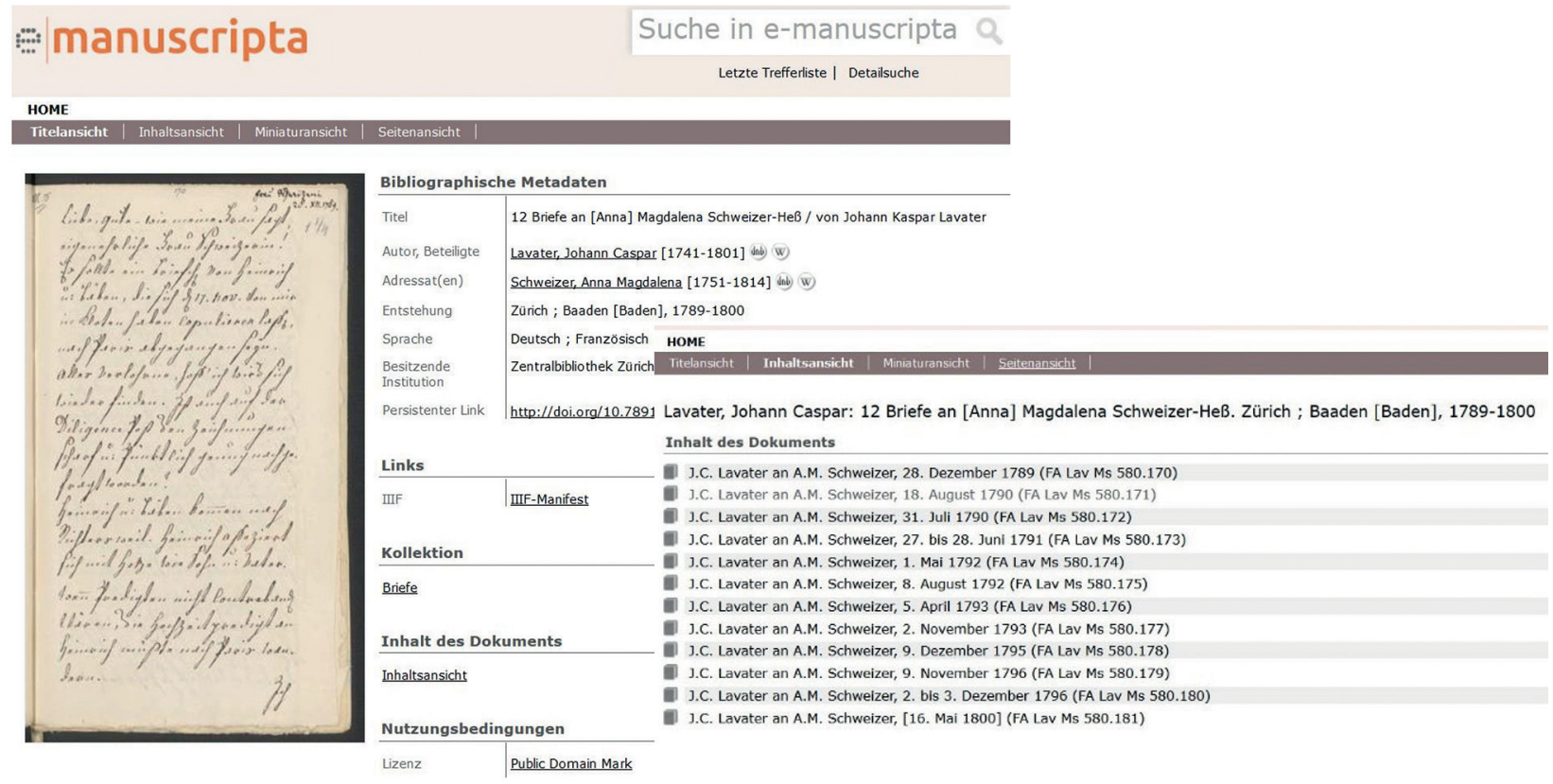

Abb. 2: Beispiel eines Briefkonvoluts mit Einzelbriefverzeichnung auf e-manuscripta (Scan und Erschließung: Zentralbibliothek Zürich)

(unterste Schicht in der Visualisierung in Abb. 1). ${ }^{15}$ Gefördert wird dieses nationale Projekt NIE-INE, das an der Universität Basel angesiedelt ist und Ende 2020 auslaufen wird, über Bundesgelder. Es handelt sich dabei um ein Förderprogramm von swissuniversities, der Rektorenkonferenz der Schweizer Hochschulen. In der Schweiz unterstützt der Bund auf Basis des Hochschulförderungsgesetzes aus dem Jahr 2015 (HFKG) die Hochschulen mit Grundbeiträgen, aber auch mit sogenannt projektgebundenen Beiträgen. Der Verwendungszweck dieser projektgebundenen Bundesmittel richtet sich auf hochschulpolitische Aufgaben von gesamtschweizerischer Bedeutung. Über die Inhalte und Programme, die mit diesem Förderinstrument unterstützt werden, entscheidet der Hochschulrat der Schweizer Hochschulkonferenz (SHK). Für die Durchführung der von der SHK beschlossenen Programme ist die Rektorenkonferenz der Schweizer Hochschulen, swissuniversities, betraut. Eines dieser Programme ist auf „Wissenschaftliche Information“ ausgerichtet und hat seit dem Start im Jahr 2013 zum Ziel, das Angebot an digitalen wissenschaftlichen Inhalten zu verbessern und optimierte Werkzeuge für deren Verarbeitung zu schaffen. ${ }^{16}$ Nachdem mit dem SNF-Call die Langzeitarchivierung für die Editionen explizit auf die Agenda der Hochschulen gekommen war, bot sich dieses Förderinstrument mit dem Programm
„Wissenschaftliche Information“ an, national und vorerst im Modus der Projektfinanzierung Lösungen anzugehen.

Die vier vom SNF geförderten Zürcher Editionen, um die es in diesem Artikel geht, partizipierten von Anfang an am Projekt NIE-INE. Die Teilnahme war alternativlos, zumal für die vom SNF geforderte Langzeitarchivierung weder national noch lokal Lösungen zur Verfügung standen. Die UZH nahm zwar bereits am nationalen Projekt Data Life Cycle Management (DLCM) teil, das die Langzeitarchivierung von Forschungsdaten aus allen Disziplinen und Fachgebieten zum Ziel hatte. ${ }^{17}$ Für die Anforderungen der langfristigen Benutzbarkeit von Editionen als Forschungsgrundlage für Dritte bestand jedoch eine Lücke, die NIEINE schließen sollte, zumal das Data and Service Center for Humanities (DaSCH) in Basel sich erst im Aufbau befand. Vorgesehen war allerdings von Anfang an, dass sich das Projekt NIE-INE technisch mit dem DaSCH abstimmt, damit sichergestellt ist, dass das Projekt NIE-INE nach Abschluss der Förderperiode 2020 in eine Hochschule oder andere Institution integriert werden kann. Das DaSCH seinerseits wird ab 2021 direkt vom SNF finanziert und mit der Langzeitarchivierung mandatiert, womit diese nationale Institution gemäß jetzigem Wissensstand prädestiniert sein wird, die Verantwortung für die Langzeitarchivierung der digitalen Editionen zu übernehmen.

15 NIE-INE steht für Nationale Infrastruktur für Editionen bzw. Infrastructure Nationales pour les Editions: https://www.nie-ine.ch/. 16 Vgl. dazu ausführlich Schneider (2018) 80 ff. sowie https://doi. org/10.1515/9783110553796-003.

17 https://www.dlcm.ch. 


\section{Fazit und Ausblick}

Es war zentrales Anliegen des SNF-Calls, die Bildung von neuen Netzwerken und geeigneten Strukturen für Forschungsprojekte wie die digitalen Editionen zu initiieren, vor allem auch mit dem Ziel der Sicherstellung der nachhaltigen Verfügbarkeit. Damit wurde auch national die Diskussion um die Koordination von Verantwortlichkeiten und Finanzierungszuständigkeiten angestoßen. Die vom SNF geförderten Editionen haben die Entwicklungen der letzten drei Jahre tiefgreifend mitgeprägt und gestaltet, sowohl lokal am Hochschulplatz Zürich als auch national. Die Editionen sind im laufenden Jahr 2020 aufgefordert, ihre Verlängerungsgesuche für die Förderperiode 20212024 einzureichen. Auch auf diesem Hintergrund ist es angezeigt, ein erstes Fazit und eine Bestandsaufnahme erreichter Ziele und noch offener Fragen zu ziehen. Erwähnenswert ist in diesem Zusammenhang zudem, dass die Förderzuständigkeit für JCLB gemeinsam mit sieben weiteren Editionen vom SNF an die Schweizerische Akademie der Geistes- und Sozialwissenschaften (SAGW) übergeben wurde. Es handelt sich dabei um langfristige Editionen, deren geplante Laufzeit auch über die Förderperiode 2021-2024 hinausreichen wird.

Wo stehen die digitalen Editionen also? Welche neu aufgebauten Strukturen haben sich bewährt? Welche Fragen sind nach wie vor ungelöst? Als gelungen kann der Aufbau der Zusammenarbeit auf lokaler Ebene bezeichnet werden. Die Schnittstellen zwischen der Bibliothek und der S3IT sind sowohl organisatorisch-inhaltlich als auch auf technischer Ebene definiert. Die Zusammenarbeit funktioniert, die Workflows sind entsprechend etabliert: Die kontinuierliche Restaurierung und Digitalisierung in der ZB wurde mit zusätzlichen Ressourcen der ZB für das Forschungsprojekt JCLB optimiert, die Metadatenerfassung auf Ebene der Einzelbriefe wird über die vom SNF finanzierte Stelle sichergestellt. Die Gesamtkoordinationsaufgabe, die ursprünglich von der ZB wahrgenommen worden war, wurde infolge der Gründung einer eigens dafür zuständigen, neuen Abteilung an die Hauptbibliothek der UZH übergeben. Zudem hat sich die Aufgabe und Rolle des Toolkoordinators, der bedarfsorientiert die einzelnen Editionen betr. geeigneter Software und mit Blick auf möglichst standardisierte technische Lösungen berät, bewährt und als unabdingbar erwiesen. Die durch diese Stelle geschaffenen Synergie- und Standardisierungseffekte über die vier geförderten Editionen hinweg sind zweifellos für die geforderte Nachhaltigkeit zentral.

Ein wichtiges Desiderat bleibt die nachhaltige Verfügbarkeit nach Ablauf der projektbasierten Förderung der digitalen Editionen. Dabei sind nicht nur die technischen
Herausforderungen der Nachhaltigkeit teilweise ungelöst, wenngleich diese zugegebenermaßen anspruchsvoll bleiben. Für digitale Editionen liegt die Problematik der Nachhaltigkeit nicht nur in der Sicherung der Primärdaten wie für viele digitale Forschungsprojekte anderer Disziplinen. Lösungen dafür bestehen bereits international, und auch national laufen entsprechende Projekte, wie das oben erwähnte DLCM an der Universität Genf oder SWISSUBase, das von FORS, dem Schweizer Kompetenzzentrum für Sozialwissenschaften, verantwortet wird. ${ }^{18}$ Die große Herausforderung für die digitalen Editionen liegt im dauerhaften Betrieb der Präsentationsoberfläche und der Aufrechterhaltung der entsprechenden Such- und Visualisierungsfunktionalitäten. Neben den technischen Herausforderungen ist jedoch weiterhin die Frage der organisatorischen Verankerung und der institutionellen Zuständigkeit auf der Agenda. Nun hat zwar der SNF im Frühling 2019 in seinem Mehrjahresprogramm 2021-2024 angekündigt, dass die Förderung von Dateninfrastrukturen und -dienstleistungen neu ins Förderprogramm aufgenommen werde. Darunter fällt - und wird auch explizit genannt - das für die digitalen Editionen relevante Data and Service Center for the Humanities (DaSCH). ${ }^{19}$ Inwiefern allerdings im Rahmen dieser Förderung des DaSCH durch den SNF die Resultate und Ergebnisse des von swissuniversities anschubfinanzierten Projekts für eine nationale Plattform für digitale Editionen (NIE-INE) übernommen werden, ist dennoch ungeklärt. Es wird wichtig sein, Kontinuität zu gewährleisten und den Zugang für die Forschenden der UZH zu nationalen und internationalen Infrastrukturen wie z. B. DARIAH sicherzustellen. ${ }^{20}$ Ob dafür z. B. an der UZH auch die Einrichtung eines DaSCH-Satelliten in Betracht gezogen werden könnte, wie dies bereits an der Universität Lausanne geschehen ist, ist eine zu diskutierende Option. ${ }^{21}$ Perspektiven für die digitalen Geisteswissenschaften im Allgemeinen sowie für die digitalen Editionen im Besonderen in Fragen der technischen Möglichkeiten und Infrastrukturen sind auch die Basis und Voraussetzung, um an der laufenden wissenschaftsimmanenten Diskussion der

\footnotetext{
$18 \mathrm{https} / /$ swissubase.ch/de/.

19 Siehe dazu das SNF-Mehrjahresprogramm, insbesondere S. 6: http://www.snf.ch/SiteCollectionDocuments/mehrjahresprogramm_ 2021_2024_d.pdf.

20 DARIAH (Digital Research Infrastructure for the Arts and Humanities) ist ein europäisches Netzwerk von digitalen Forschungsinfrastrukturen für die Geistes- und Kulturwissenschaften. Die Schweiz ist nicht Mitglied von DARIAH, allerdings sind sieben Universitäten sowie die SAGW Kooperationspartner von DARIAH: https://sagw.ch/ sagw/community/internationale-beziehungen/dariah/dariah-vertret ungen-an-ch-unis/.

21 https:/platec.unil.ch/.
} 
eigenen Rolle und Positionierung partizipieren zu können. Denn:

\begin{abstract}
„Der ganze Prozess ist immer noch vom Basteln und Ausprobieren gekennzeichnet, nicht zuletzt weil gerade in den letzten ein bis zwei Jahren die digitalen Analysemethoden für Texte noch einmal erheblich verbessert wurden. Mit welchen digitalen Methoden welche interessanten Ergebnisse für die Geistes- und Kulturwissenschaften ermittelt werden können, ist eine weitgehend offene Frage. Aufgrund der bisherigen Ergebnisse und mit Blick auf die neu entstehenden Datensammlungen in den Bibliotheken und Archiven darf man durchaus optimistisch sein, zumindest aber sehr neugierig." 22
\end{abstract}

Der Erfolg der vier an der UZH kooperierenden digitalen Editionsprojekte hat denn auch ein weiteres Desiderat aufgezeigt. Trotz fehlender organisatorischer Verankerung der digitalen Geisteswissenschaften an der UZH, arbeiten die vier vom SNF geförderten Editionen erfolgreich zusammen. Sie treffen sich regelmäßig und tauschen sich über best practice und geeignete technische Umsetzungen aus. Ankerpunkt dieses Netzwerks an der UZH ist die befristete und vom SNF finanzierte Stelle des Toolkoordinators in der Science-IT Abteilung, der S3IT. Doch über diese technische Koordination hinaus fehlt es an einer Plattform für einen genuin wissenschaftsimmanenten Austausch. Dazu kommt, dass weit mehr als die vier vom SNF geförderten digitalen Editionen an der UZH aktiv sind; und dies fakultätsübergreifend vor allem an der Philosophischen, aber auch an der Theologischen und der Rechtswissenschaftlichen Fakultät. Aktuell laufen deshalb an der UZH Gespräche, ob bzw. wie alle digitalen Editionsprojekte an der UZH im Rahmen eines Kompetenznetzwerkes gestärkt und sichtbar gemacht werden könnten.

Vieles ist erreicht, einiges noch offen: Die digitalen Editionen können mittlerweile auf ein funktionierendes Netzwerk an konkreten Dienstleistungen im Bereich der Digitalisierung, der Metadatenverzeichnung sowie der technischen Umsetzung ihrer Editionen zurückgrei- fen. Desiderate bleiben verstärkt wissenschaftsgetriebene Kompetenznetzwerke sowie der Zugang zu nationalen und internationalen, für die Nachhaltigkeit relevanten Infrastrukturen.

\section{Literaturverzeichnis}

Aschenbrenner, Andreas; Neuroth, Heike (2011): ForschungsdatenRepositorien. In: Handbuch Forschungsdatenmanagement, hg. v. Stephan Büttner, Hans-Christoph Hobohm und Lars Müller. Bad Honnef: Bock und Herchen.

Jannidis, Fotis (2019): Digitale Geisteswissenschaften: Offene Fragen - schöne Aussichten. In: Zeitschrift für Medien-und Kulturforschung, 10 (1), 70.

Schneider, Gabi (2018): Das Programm P-5 „Wissenschaftliche Information: Zugang, Verarbeitung und Speicherung“: teilnehmeroffene Dienste der Hochschulen, für die Hochschulen. In: Bibliotheken der Schweiz: Innovation durch Kooperation: Festschrift für Susanna Bliggenstorfer anlässlich ihres Rücktrittes als Direktorin der Zentralbibliothek Zürich, hg. v. Alice Keller und Susanne Uhl. Berlin: De Gruyter, 78-96.

RfIl (2016): RfII - Rat für Informationsinfrastrukturen, Leistung aus Vielfalt. Empfehlungen zu Strukturen, Prozessen und Finanzierung des Forschungsdatenmanagements in Deutschland, Göttingen. Verfügbar unter http://www.rfii.de/download/rfiiempfehlungen-2016/.

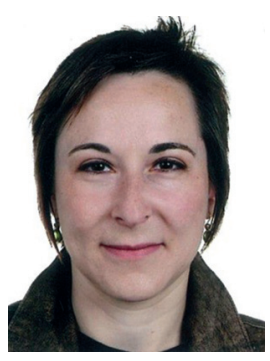

\section{Dr. Andrea Malits}

Hauptbibliothek der Universität Zürich

Strickhofstr. 39

$\mathrm{CH}-8057$ Zürich

Schweiz

andrea.malits@hbz.uzh.ch 